\title{
Flow over a Finite Forchheimer Porous Layer with Variable Permeability
}

\author{
M.H. Hamdan ${ }^{1}$, M.S. Abu Zaytoon ${ }^{2}$ \\ ${ }^{1,2}$ Department of Mathematics and Statistics, University of New Brunswick, Saint John, N.B., Canada, E2L 4L5
}

\begin{abstract}
The problem of flow through a Navier-Stokes channel overlying a finite Forchheimer porous layer of variable permeability is considered. Choices of permeability distributions that will bring the Forchheimer velocity to zero on the bounding solid wall are discussed and their influence on the resulting velocity profile in the Navier-Stokes channel and on the slip velocity are analyzed.

Keywords: Forchheimer equation, Variable Permeability, Slip velocity
\end{abstract}

\section{Introduction}

Flow over porous layers is driven by various industrial applications including lubrication mechanisms involving porous plates and porous linings, design of heating and cooling systems, engine cooling systems, and the design of porous surfaces (such as aircraft wings with porous cavities) to reduce the drag (cf. [1], [2] and the references therein). In addition, studies of fuel cells heavily depend on analysis of flow through and over porous layers. These and many other applications, together with a literature review of what has been accomplished in this field, have been discussed in greater details, [2], [3], [4].

Analysis of coupled parallel flow involves the study of flow through a free-space channel underlain by a porous layer of either finite or infinite depth with an interface region between a porous medium and a fluid where momentum and mass transfer take place. Flow through the (free-space) channel is governed by NavierStokes equations, namely the following equation written here for unidirectional flow:

$\frac{d^{2} u}{d y^{2}}=\frac{1}{\mu} \frac{d p}{d x}$

where $u$ is the velocity in the channel, $\mu$ is the viscosity, $p$ is the pressure, and $d p / d x<0$ is the driving pressure gradient.

In the porous layer, the flow is governed by an appropriate model, although the use of Darcy's law in the porous sediment has been predominant. Darcy's equation is written here for unidirectional flow as:

$\frac{d p}{d x}+\frac{\mu}{k} u_{D}=0$

where $u_{D}$ is the uniform Darcy velocity in the porous layer and $k$ is the constant permeability,

Coupled parallel flow gained interest following the experiments of Beavers and Joseph, [5], to determine the matching conditions at the interface between a porous medium and free-space. With their experimental observation that the mass flux through free-space channel is larger than that predicted by the Poiseuille flow when a no-slip condition is imposed, Beavers and Joseph, [5], provided an explanation in terms of a slip flow hypothesis at the interface $y=0$ and proposed the following empirical slip-flow condition that agreed well with their experiments:

$$
\frac{\partial u}{\partial y} \mid\left(x, 0^{+}\right)=\frac{\alpha}{\sqrt{k}}\left[u\left(x, 0^{+}\right)-u_{D}\right]
$$

where $u\left(x, 0^{+}\right)$is the tangential velocity in the free-space channel and $\alpha$ is a slip coefficient that depends on the porous medium properties. Nield and Bejan [1] provided the range of 0.01 to 5 and reported that Beavers and Joseph [5] used the following values for $\alpha: 0.78,1.45$, and 4.0 for Foametal having average pore sizes $0.016,0.034$, and 0.045 inches, respectively, and 0.1 for Aloxite with average pore size 0.013 or 0.027 inches.

Condition (3) is also used when the flow through an infinite porous layer is governed by the following Forchheimer's equation, written here for unidirectional flow:

$\frac{d p}{d x}+\frac{\mu}{k} v+\frac{\rho C_{f}}{\sqrt{k}} v|v|=0$ 
where $\rho$ is the fluid density, $v$ is the Forchheimer velocity, and $C_{f}$ is the Forchheimer drag coefficient.

Equations (2) and (4) have been used in connection with coupled parallel flow over an infinite porous layer with constant permeability. These equations are marked with the absence of a viscous shear term that is important in the presence of a solid wall that bounds a finite porous layer, and on which a no-slip condition is imposed. This situation motivates the current work in which we consider parallel flow over a finite porous layer bounded by an impermeable wall. In order to impose a no-penetration condition on the wall and to bring the velocity to zero, we introduce a variable permeability function that falls to zero on the wall, and in terms of which the velocity in the finite layer is defined. When the permeability is zero, the velocity will fall to zero. This approach has been successfully implemented in the case of modelling coupled parallel flow over a Darcy layer with variable permeability [6]. The current work deals with a Forchheimer finite porous layer with variable permeability. Fluid flow through porous media with variable permeability enjoys many natural and industrial applications, and has been extensively studied, (cf. [7-11] and the references therein).

For the case of constant permeability, the velocity profile in an infinite porous layer is constant, while the velocity in the channel is a quadratic function. At the interface, the velocity in the channel is higher than the velocity in the layer. Velocity continuity necessitates the concept of the slip hypothesis and the Beavers-Joseph condition. This slip velocity is quantified in this work for variable permeability and finite porous layer of the Forchheimer type.

\section{II.1. Problem Formulation}

\section{Problem Formulation and Solution}

Consider the Navier-Stokes flow through a channel of depth $h$ bounded by a Forchheimer porous layer of finite thickness possessing variable permeability, as shown in Fig. 1. The interface between the channel and the porous layer is assumed to be a sharp interface along $\mathrm{y}=0$.

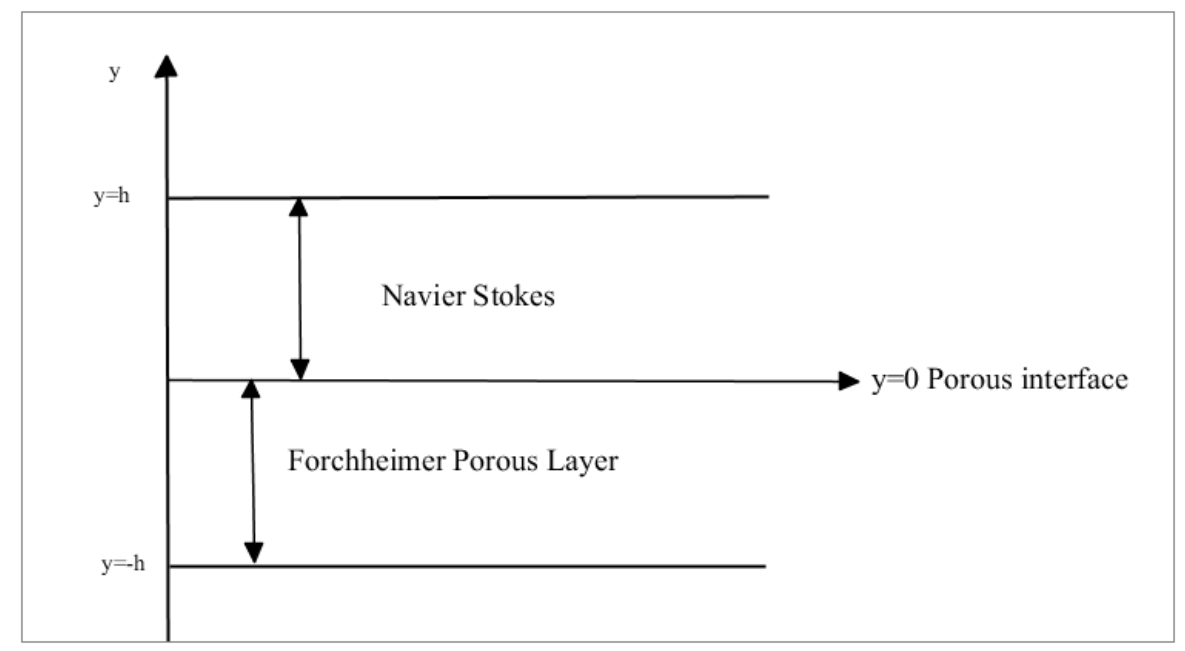

Fig. 1: Diagramatic Sketch of a Channel Bounded by a Porous Layer

Flow in the channel is governed by Navier-Stokes equation (1) and flow through the porous layer is governed by the Forchheimer's equation (4). Flow through the given configuration is assumed to be driven by the same constant pressure gradient.

Governing equations (1) and (4) take the following dimensionless forms in the channel and in the Forchheimer porous layer, respectively, wherein $P_{X}=d P / d X$ :

$$
\frac{d^{2} U}{d Y^{2}}=\operatorname{Re} P_{X}
$$

$\operatorname{Re} P_{X}+\frac{V}{K}+\frac{\operatorname{Re} C_{f}}{\sqrt{K}} V^{2}=0$

wherein we have used the following convenient dimensionless quantities

$$
(X, Y)=\frac{(x, y)}{h} ;(U, V)=\frac{(u, v)}{u_{c}} ; K=\frac{k}{h^{2}} ; P=\frac{p}{\rho\left(u_{c}\right)^{2}} ; \operatorname{Re}=\frac{\rho u_{c} h}{\mu} .
$$


Dimensionless channel and porous layer extents are thus given, respectively, by $0<Y<1$ and $-1<Y<0$. The sharp interface is located at $Y=0$, and the upper and lower bounding walls are at $Y=1$ and $Y=-1$, respectively.

\section{II.2. Problem Solution}

It is required to solve (5) and (6) subject to the conditions of no-penetration and the vanishing of permeability on the solid, lower boundary; a no-slip condition on the upper boundary; and the Beavers and Joseph condition at the interface between the channel and the porous layer.

In the absence of viscous shear effects in the Forchheimer equation, the vanishing of velocity $V$ on the lower boundary can be seen from equation (6) by multiplying both sides by $K$, and taking $K=0$ on the lower boundary. This velocity varies in the Forchheimer porous layer due to variations in permeability. The dimensionless permeability $K(Y)$ increases from 0 on the lower boundary to reach its maximum $k_{\max }$ at the interface, $Y=0$.

Dimensionless boundary and interfacial conditions are thus stated as follows:

$$
\begin{aligned}
& U(1)=0=V(-1) \\
& K(-1)=0 \\
& K(0)=k_{\max } \\
& U(0)=U_{i}
\end{aligned}
$$

and condition (3) is written in the following dimensionless form for Forchheimer's case:

$\frac{d U}{d Y}=\frac{\alpha}{\sqrt{K}}\left(U_{i}-V\right)$ at $Y=0$.

Now, equation (6) is an algebraic equation for the Forchheimer velocity $V(Y)$ whose non-negative solution is given by:

$$
V=-\frac{1}{2 \operatorname{Re} C_{f} \sqrt{K}}\left[1-\sqrt{1-4 \operatorname{Re}^{2} C_{f} K \sqrt{K} P_{X}}\right] .
$$

Forchheimer velocity at the interface is expressed in terms of $k_{\max }$ and is given by:

$$
V(0)=-\frac{1}{2 \operatorname{Re} C_{f} \sqrt{K_{\max }}}\left[1-\sqrt{1-4 \operatorname{Re}^{2} C_{f} K_{\max } \sqrt{K_{\max }} P_{X}}\right] .
$$

Equation (5) yields the following general solution for the Navier-Stokes velocity in the channel:

$U=\operatorname{Re} P_{X} \frac{Y^{2}}{2}+c_{1} Y+c_{2}$

where $c_{1}, c_{2}$ are arbitrary constants that take the following forms when conditions (8) are implemented:

$$
\begin{aligned}
& c_{1}=\frac{\alpha}{\sqrt{k_{\max }}}\left[U_{i}+\frac{1}{2 \operatorname{Re} C_{f} \sqrt{k_{\max }}}\left(1-\sqrt{1-4 \operatorname{Re}^{2} C_{f} k_{\max } \sqrt{k_{\max }} P_{X}}\right)\right] \\
& c_{2}=-\frac{1}{2} \operatorname{Re} P_{X}-\frac{\alpha}{\sqrt{k_{\max }}}\left[U_{i}+\frac{1}{2 \operatorname{Re} C_{f} \sqrt{k_{\max }}}\left(1-\sqrt{1-4 \operatorname{Re}^{2} C_{f} P_{X} k_{\max } \sqrt{k_{\max }} P_{X}}\right)\right] .
\end{aligned}
$$

Solution to (5) satisfying conditions (8) can thus be written as:

$$
U=\frac{\operatorname{Re} P_{X}}{2}\left(Y^{2}-1\right)+\frac{\alpha}{\sqrt{k_{\max }}}\left[U_{i}+\frac{1}{2 \operatorname{Re} C_{f} \sqrt{k_{\max }}}\left(1-\sqrt{1-4 \operatorname{Re}^{2} C_{f} P_{X} k_{\max } \sqrt{k_{\max }} P_{X}}\right)\right](Y-1) .
$$

From (14) we obtain

$$
U(0)=-\frac{\operatorname{Re} P_{X}}{2}-\frac{\alpha}{\sqrt{k_{\max }}}\left[U_{i}+\frac{1}{2 \operatorname{Re} C_{f} \sqrt{k_{\max }}}\left(1-\sqrt{1-4 \operatorname{Re}^{2} C_{f} P_{X} k_{\max } \sqrt{k_{\max }} P_{X}}\right)\right]
$$


and upon using condition (8d) in (14), and subsequently solving for $U_{i}$, we obtain

$U_{i}=-\frac{\sqrt{k_{\max }} \operatorname{Re} P_{X}}{2\left[\sqrt{k_{\max }}+\alpha\right]}\left[1+\frac{4 \alpha \sqrt{k_{\max }}}{\left(1+\sqrt{1-4 k_{\max } \sqrt{k_{\max }} C_{f} \operatorname{Re}^{2} P_{X}}\right)}\right]$.

Velocity distribution (14) in the channel can then be expressed in the form

$U=\frac{\operatorname{Re} P_{X}}{2}\left(Y^{2}-1\right)+\alpha\left[\frac{U_{i}}{\sqrt{k_{\max }}}+\frac{2 \operatorname{Re} P_{X} \sqrt{k_{\max }}}{\left(1+\sqrt{1-4 \operatorname{Re}^{2} C_{f} k_{\max } \sqrt{k_{\max }} P_{X}}\right)}\right](Y-1)$.

with $U_{i}$ given by (16). Using (10) and (16) we obtain the slip velocity at the interface as:

$U_{i}-V(0)=\frac{\operatorname{Re} P_{X}}{2}\left[\frac{2 k_{\max }}{1+\sqrt{1-4 k_{\max } \sqrt{k_{\max }} C_{f} \operatorname{Re}^{2} P_{X}}}-1\right]\left[\frac{\sqrt{k_{\max }}}{\left[\sqrt{k_{\max }}+\alpha\right]}\right]$.

By comparison, when the flow is through a channel over an infinite Forchheimer porous layer, velocity in the porous layer is given by equation (9) with the permeability $K$ being constant. This profile represents a uniform velocity across the infinite porous layer. Velocity in the channel in this case is given by:

$U=\frac{\operatorname{Re} P_{X}}{2}\left(Y^{2}-1\right)+\alpha\left\{\frac{U_{i}}{\sqrt{K}}+\frac{2 \operatorname{Re} P_{X} \sqrt{K}}{\left(1+\sqrt{\left[1-4 \operatorname{Re}^{2} K \sqrt{K} C_{f} P_{X}\right]}\right)}\right\}(Y-1)$

where

$U_{i}=-\frac{\sqrt{K} \operatorname{Re} P_{X}}{2[\sqrt{K}+\alpha]}\left[1+\frac{4 \alpha \sqrt{K}}{1+\sqrt{\left[1-4 K \sqrt{K} C_{f} \operatorname{Re}^{2} P_{X}\right]}}\right]$.

Clearly, if $K=k_{\max }$ then (16) and (20) give the same value for $U_{i}$, while (17) and (19) give the same velocity profile in the channel. While this is the case for constant permeability porous layer, the value of $k_{\max }$ in the case of variable permeability represents the maximum permeability reached at the interface.

\section{II.3. The Case of Velocity Continuity}

In case of velocity continuity at the interface, condition (8d) is replaced by

$U(0)=U_{i}=V(0)$.

Velocity distributions in the Navier-Stokes channel and in the Forchheimer porous layer are given, respectively, by

$$
\begin{aligned}
& U(Y)=\operatorname{Re} P_{X}\left(\frac{Y^{2}-Y}{2}\right)+\frac{1}{2 \operatorname{Re} C_{f} \sqrt{k_{\max }}}\left(1-\sqrt{1-4 \operatorname{Re}^{2} C_{f} P_{X} k_{\max } \sqrt{k_{\max }} P_{X}}\right)(Y-1) \\
& V=-\frac{1}{2 \operatorname{Re} C_{f} \sqrt{K}}\left[1-\sqrt{1-4 \operatorname{Re}^{2} C_{f} K \sqrt{K} P_{X}}\right]
\end{aligned}
$$

while the velocity at the interface takes the form

$U_{i}=U(0)=V(0)=-\frac{1}{2 \operatorname{Re} C_{f} \sqrt{K_{\max }}}\left[1-\sqrt{1-4 \operatorname{Re}^{2} C_{f} K_{\max } \sqrt{K_{\max }} P_{X}}\right]$. 


\section{Results and Discussion}

Sample results are discussed in this section for selected values of permeability, slip parameter, pressure gradient and Reynolds number.

Table 1 illustrates the slip velocity, given by equation (18), and the velocity at the interface, $U_{i}$, given by equation (16), for the case of variable permeability Forchheimer layer and shows their dependence on the slip parameter $\alpha$, the pressure gradient and Reynolds number. A decrease in $\alpha$ results in an increase in the lip velocity, as evidenced from equation (18), while increasing Re and the magnitude of pressure gradient have the expected effect of increasing the slip velocity. The increase in $U_{i}$ with increasing Re and magnitude of pressure gradient is also evident, for a given value of $\alpha$. Equation (16) demonstrates how $U_{i}$ increases with increasing $\alpha$. All values are calculated at $k_{\max }=1$.

\begin{tabular}{|l|l|l|l|l|}
\hline $\operatorname{Re}$ & $P_{X}$ & $\alpha$ & $U_{i}$ & Slip Velocity \\
\hline 1 & -1 & 1.45 & 0.6285116341 & 0.0577264597 \\
\hline 1 & -1 & 0.1 & & 0.1285725693 \\
\hline 5 & -1 & 1.45 & 1.718056135 & 0.7798398970 \\
\hline 5 & -1 & 0.1 & & 1.7369161342 \\
\hline 5 & -3 & 1.45 & 4.340032886 & 2.6202560769 \\
\hline 5 & -3 & 0.1 & & 5.8360248985 \\
\hline 10 & -3 & 1.45 & 7.451925208 & 5.6640089004 \\
\hline 10 & -3 & 0.1 & & 12.615292551 \\
\hline
\end{tabular}

Table 1. Slip and interfacial velocities for different $\operatorname{Re}, P_{X}$ and $\alpha ; k_{\max }=1$.

For the case of infinite Forchheimer layer with constant permeability, $U_{i}$ depends additionally on the value of the constant permeability. The effect of permeability on $U_{i}$ is illustrated in Table 2 for $\alpha=1.45, \operatorname{Re}=1$ and $P_{X}=-1$. Table 2 shows that $U_{i}$ decreases with decreasing permeability in the layer.

\begin{tabular}{|l|l|}
\hline$K$ & $U_{i}$ \\
\hline 1 & 0.6285116341 \\
\hline 0.1 & 0.1702362852 \\
\hline 0.01 & 0.04160776371 \\
\hline 0.001 & 0.01165030894 \\
\hline 0.0001 & 0.003523972548 \\
\hline
\end{tabular}

Table 2. Interfacial velocity for different constant medium permeability; $\operatorname{Re}=1, P_{X}=-1$.

In the case of using velocity continuity condition (21), velocity profiles (22) and (23) are sketched across the porous layer and the channel for the following variable permeability distributions

$$
\begin{aligned}
& K(Y)=k_{\text {max }}\left(1-Y^{2}\right) \\
& K(Y)=k_{\text {max }}(1+Y)^{2} \\
& K(Y)=\frac{1}{2} k_{\text {max }}\left(2+Y-Y^{2}\right) \\
& K(Y)=\frac{1}{e} k_{\text {max }}(1+Y) e^{(1+Y)} \\
& K(Y)=\frac{1}{e} k_{\text {max }}(1+Y) e^{(1-Y)} .
\end{aligned}
$$

All of these permeability distributions satisfy the variable permeability conditions (8b) and (8c), and the corresponding velocity profiles are illustrated in Figs. 2-6, respectively, for $k_{\max }=1, P_{X}=-1, \operatorname{Re}=1$, and $C_{f}=0.55$. These profiles show the absence of a slip velocity due to the fact that velocity continuity condition (21) has been used. The effect of variable permeability is to generate velocity variations in the porous layer in such a way that a no-slip condition is satisfied on the solid wall bounding the porous layer. Velocity then increases nonlinearly until it matches, at the interface, the parabolic velocity distribution in the Navier-Stokes channel 


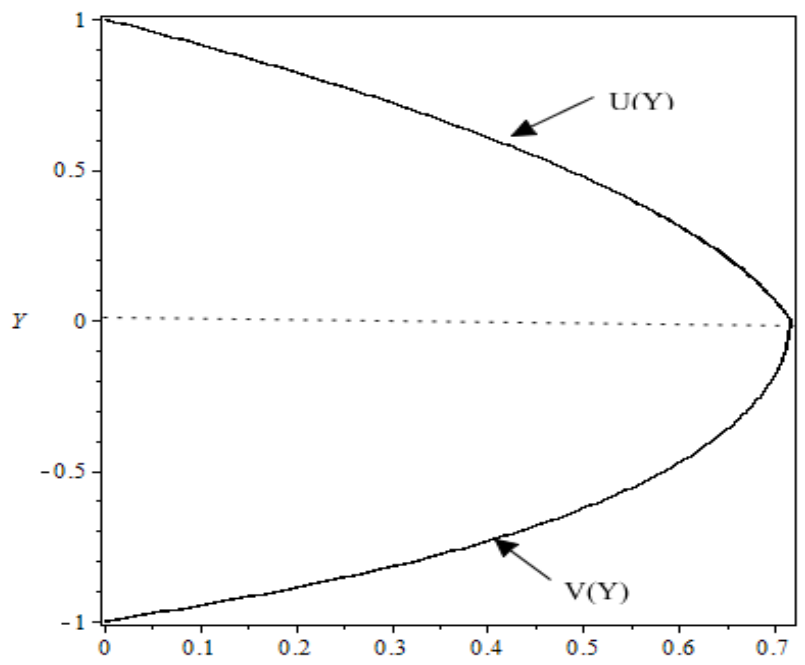

Fig. 2. Velocity profiles across the flow configuration.

$K(Y)=k_{\max }\left[1-Y^{2}\right], k_{\max }=1, P_{X}=-1, \operatorname{Re}=1, C_{f}=0.55$.

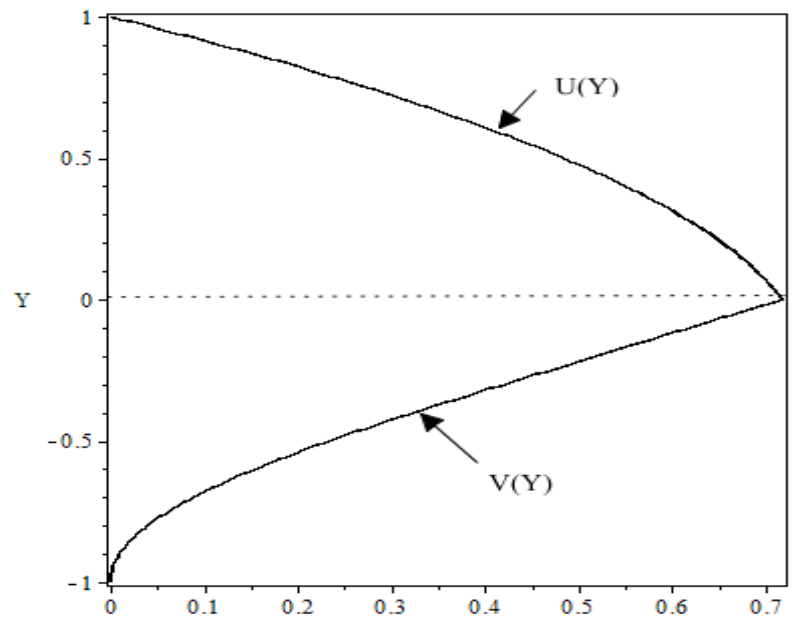

Fig. 3. Velocity profiles across the flow configuration.

$K(Y)=k_{\text {max }}(1+Y)^{2}, k_{\text {max }}=1, P_{X}=-1, \operatorname{Re}=1, C_{f}=0.55$.

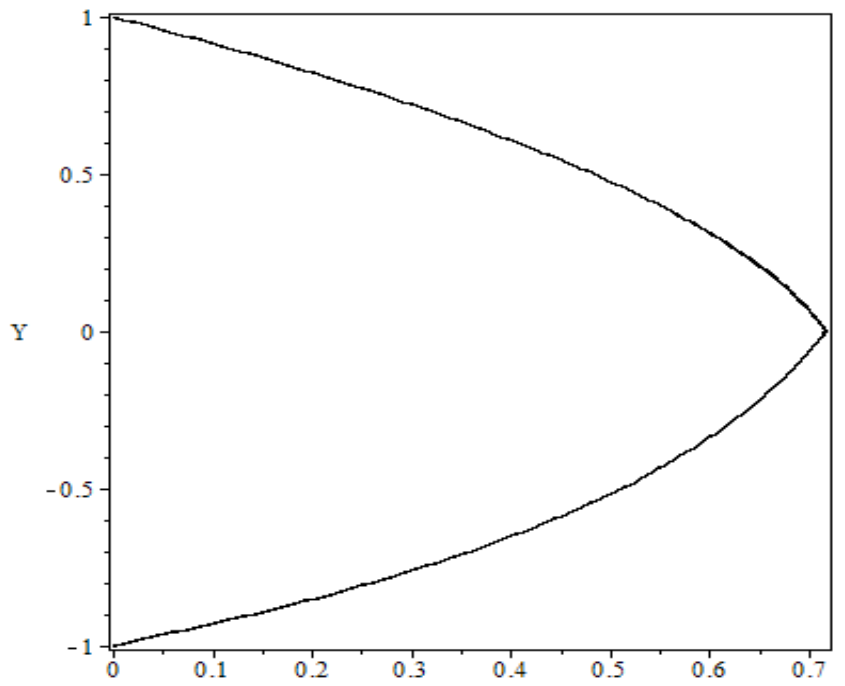

Fig. 4. Velocity profiles across the flow configuration. 


$$
K(Y)=\frac{1}{2} k_{\max }\left(2+Y-Y^{2}\right), k_{\max }=1, P_{X}=-1, \operatorname{Re}=1, C_{f}=0.55
$$

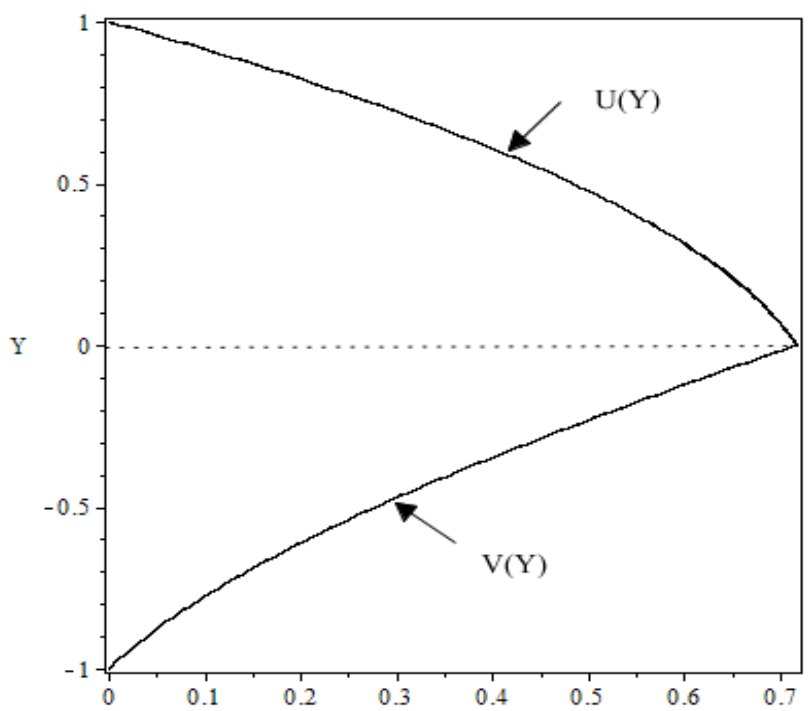

Fig. 5. Velocity profiles across the flow configuration.

$$
K(Y)=\frac{1}{e} k_{\text {max }}(1+Y) e^{(1+Y)}, k_{\max }=1, P_{X}=-1, \operatorname{Re}=1, C_{f}=0.55 .
$$

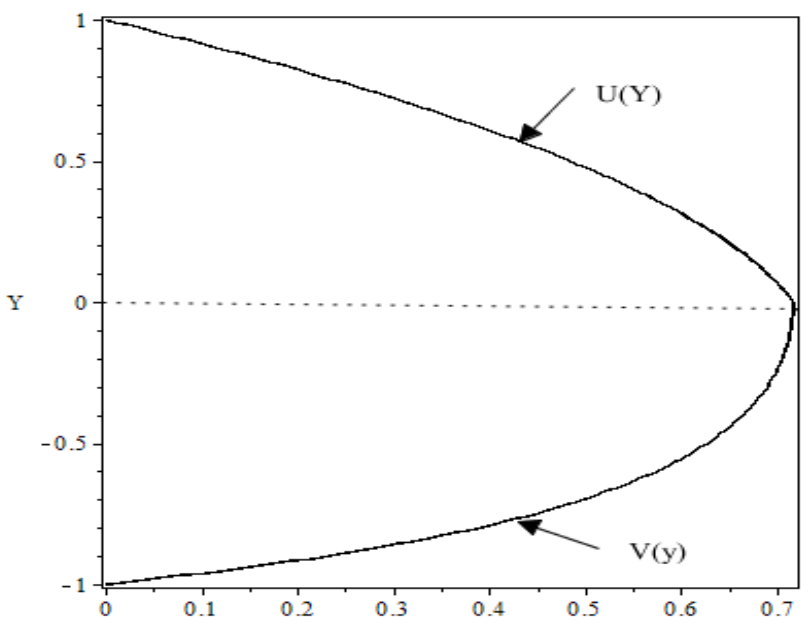

Fig. 6. Velocity profiles across the flow configuration.

$$
K(Y)=\frac{1}{e} k_{\max }(1+Y) e^{(1-Y)}, k_{\max }=1, P_{X}=-1, \operatorname{Re}=1, C_{f}=0.55 .
$$

\section{Conclusion}

In this work we considered the coupled, parallel flow through a channel bounded by a porous layer of finite thickness. Flow in the porous layer is assumed to be governed by Forchheimer's equation. Although this equation is not compatible with the imposition of a no-slip condition in the presence of a solid boundary, such a condition is facilitated by the introduction of variable permeability in the porous layer. The no-penetration, zero permeability on the boundary generates a zero fluid velocity there. The velocity distributions in the channel and porous layer, the velocity $U_{i}$ at the interface and the slip velocity at the interface have been determined for variable and constant permeability Forchheimer layer and are shown to depend on the pressure gradient, Reynolds number, the value of $k_{\max }$, and the Forchheimer drag coefficient. With the exception of velocity in the Forchheimer layer, velocities are dependent on the Beavers and Joseph slip parameter. Velocity in the 
porous layer depends on the permeability distribution, $K(Y)$. Suitable permeability distributions must fall to zero on the lower boundary and reach $k_{\max }$ at the interface.

\section{References}

[1]. D.A. Nield and A. Bejan, Convection in Porous Media, $\left(5^{\text {th }}\right.$ ed. Springer, 2017).

[2]. R.A. Ford, M.S. Abu Zaytoon and M.H. Hamdan, Simulation of flow Through Layered Porous Media IOSR Journal of Engineering, 6(6), 2016, pp. 13-17.

[3]. K. Vafai and R. Thiyagaraja, Analysis of flow and heat transfer at the interface region of a porous medium, Int. J. Heat Mass Transfer, 30(7), 1987, 1391-1405.

[4]. B. Alazmi and K. Vafai, Analysis of fluid flow and heat transfer interfacial conditions between a porous medium and a fluid layer, International Journal of Heat and Mass Transfer, 44, 2001, 1735- 1749.

[5]. G.S. Beavers and D.D. Joseph, Boundary conditions at a naturally permeable wall, Journal of Fluid Mechanics, $30,1967,197-207$.

[6]. M.S. Abu Zaytoon, T.L. Alderson and M. H. Hamdan, Flow over a Darcy porous layer of variable permeability, Journal of Applied Mathematics and Physics, 4, 2016, 86-99.

[7]. D. A. Nield and A. V. Kuznetsov, The effect of a transition layer between a fluid and a porous medium: shear flow in a channel, Transport in Porous Media,78, 2009, 477-487.

[8]. M.S. Abu Zaytoon, T.L. Alderson and M.H. Hamdan, Flow through a layered porous configuration with generalized variable permeability, Int. J. of Enhanced Research in Science, Technology \& Engineering, 5(6), 2016, 1-21.

[9]. M.S. Abu Zaytoon, T.L. Alderson and M. H. Hamdan, Flow through a Variable Permeability Brinkman Porous Core, Journal of Applied Mathematics and Physics, 4, 2016, 766-778.

[10]. M.S. Abu Zaytoon, T.L. Alderson and M. H. Hamdan, Flow through layered media with embedded transition porous layer, , Int. J. of Enhanced Research in Science, Technology \& Engineering, 5(4), 2016, 9-26.

[11]. M.S. Abu Zaytoon, T.L. Alderson and M. H. Hamdan, Flow through variable permeability composite porous layers, Gen. Math. Notes, 33(1), 2016, 26-39. 